\title{
Personalentwicklung in sechs Schritten
}

\author{
Inken Schubert
}

Personalentwicklung ist auch für kleine Träger in der Sozialwirtschaft erforderlich - und machbar. Es muss dabei nicht alles sofort passieren: in sechs Schritten kann ein auf die jeweilige Einrichtung zugeschnittenes Konzept erarbeitet und umgesetzt werden.

Was Unternehmen der freien Wirtschaft schon lange systematisch durchführen - angefangen bei Fortbildungen für einzelne Mitarbeiter bis hin zum strategisch relevanten »Human Ressource Management «, ist in vielen Einrichtungen und Organisationen des sozialen Sektors noch nicht richtig angekommen: systematische Personalentwicklung als wirksames Mittel zur Sicherung des Unternehmenserfolges. "Systematisch « meint dabei die wiederholte, regelhafte Durchführung von Maßnahmen der Personalentwicklung. Nichts hindert auch kleine Einrichtungen der Sozialwirtschaft, sich etwas von den "Großen « der gewerblichen Wirtschaft abzuschauen.

Nach mehreren Jahren eigener Erfahrung - zunächst in der Personalentwicklung eines Versicherungskonzerns, dann als ehrenamtlicher Personalvorstand in einem Kindergarten - komme ich zu dem Schluss, dass es vor allem drei Glaubenssätze sind, die ein beherztes Angehen des Themas in kleinen Sozialeinrichtungen verhindern:

1. »Das brauchen wir nicht. Ab und zu eine Fortbildung, das hat bisher immer gereicht.«

2. »Das schaffen wir nicht. Dafür haben wir keine Leute, kein Geld und keine Zeit!«

3. »Bei uns ist doch alles anders als in der freien Wirtschaft. Das kann man gar nicht übertragen!«

Auch wenn es bei sozialen Diensten und Einrichtungen wirklich oft ganz anders läuft als in gewinnorientierten Konzernen, setze ich heute drei Behauptungen dagegen:

1. Es ist vorteilhaft und dringend notwendig, auch in kleinen Einrichtungen eine systematische Personalentwicklung durchzuführen.

2. Man muss nicht alles auf einmal machen. Systematische Personalentwicklung kann Schritt für Schritt erfolgen.

3. Man muss Personalentwicklung nicht wie ein großes Wirtschaftsunternehmen betreiben, aber das Grundanliegen der Personalentwicklung ist bei Großen wie Kleinen das Gleiche. Daher kann man durchaus vieles übertragen.

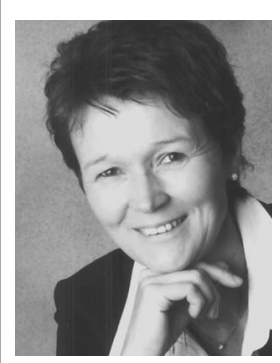

Inken Schubert (44) hat Wirtschaftspädagogik an der Universität Hamburg studiert. Nach langjährigen Erfahrungen im Personalbereich als Ausbildungsleiterin und in der Personalentwicklung und mehreren Jahren als ehrenamtlicher Personalvorstand in einem Waldorf-Kindergarten ist sie derzeit tätig für Niederholz \& Partner, einem Personal- und Organisationsberatungsunternehmen in Hamburg. inkenschubert@aol.com

\section{»Das brauchen wir nicht! Oder doch?«}

Für kleine Einrichtungen gilt, was auch die großen Unternehmen längst wissen: Das wichtigste Kapital sind die Mitarbeiterinnen und Mitarbeiter. Dass sie adäquat ausgebildet, motiviert und engagiert an der richtigen Stelle des Unternehmens ihre Arbeit tun, ist einer der wichtigsten Erfolgsfaktoren für den Betrieb. Und gerade in sozialen Arbeitszusammenhängen, in denen die Beziehungen der Menschen zueinander von zentraler Bedeutung sind, steht und fällt der Erfolg mit den persönlichen Qualifikationen der Mitarbeitenden. Ihre Persönlichkeit, ihre Kreativität, ihre Motivation sind wichtiger als der Einsatz neuester Techniken. Hier kann Personalentwicklung einen wichtigen Beitrag zur Qualifizierung der Mitarbeiterinnen und Mitarbeiter leisten.

Die Arbeitswelt ändert sich laufend. Das gilt auch für soziale Einrichtungen: Sozialpolitische, gesellschaftliche und wirtschaftliche Gegebenheiten sind komplex und wandelbar, bildungspolitische Aufträge werden immer diffiziler, der Legitimationsdruck hinsichtlich Qualität und Kosten steigt. Zudem hat sich in den vergangenen Jahren das Selbstverständnis der Kunden und somit auch das der Einrichtungen stark gewandelt: aus versorgten Leistungsempfängern werden mehr und mehr souveräne Kunden, aus Versorgern werden unterstützende Dienstleister. Da braucht eine Einrichtung Fachkräfte mit profundem Wissen, die bereit und in der Lage sind, flexibel und verantwortlich zu handeln. Auch hier unterstützt eine systematische Personalentwicklung die Mitarbeiterinnen und Mitarbeiter. 
Und dabei ist Personalentwicklung für eine soziale Einrichtung noch wichtiger als für einen Wirtschaftskonzern: Einerseits ist das Funktionsspektrum der einzelnen Stellen besonders breit; andererseits ist der Personalschlüssel in der Regel eng bemessen. Das bedeutet, es kommt auf jeden an. Wenn einer seine Arbeit nicht optimal leistet, weil ihm das Wissen fehlt oder die Motivation, dann kann das niemand auffangen und es zeigen sich sofort spürbare Auswirkungen auf die Qualität der Leistung der Einrichtung. Es ist also einerseits notwendig, sich überschneidende Fähigkeitsprofile bei den Mitarbeitenden zu schaffen, andererseits ist ihre Bindung und damit die Ausfallsicherheit durch motivierende Maßnahmen zu unterstützen. Beides kann eine systematische Personalentwicklung leisten. Hier und da mal eine Fortbildung für den einen oder anderen Mitarbeiter, das reicht hier nicht.

\section{"Das schaffen wir nicht! Oder doch?«}

Mit der Idee, eine systematische Personalentwicklung einzuführen, tauchen Fragen auf, die sich viele Träger und Mitarbeitende noch nie gestellt haben: Was ist denn eigentlich das Ziel unserer Einrichtung? Woran erkennen wir, dass wir es erreicht haben? Wie wollen wir lernen und arbeiten? Was ist uns wichtig? Angesichts solch Fragestellungen kann einen schon der Mut verlassen, aber dies sind wichtige Fragen.

Es sind die Fragen, die sich die Verantwortlichen in Banken und Versicherungen, Handelsunternehmen und Industriebetrieben vor Jahrzehnten stellten, als man dort mit systematischer Personalentwicklung begann. Denn Personalentwicklung geschieht nicht im luftleeren Raum, sondern ist eingebettet in Strategie, Struktur und Kultur des Unternehmens. Große soziale Einrichtungen und Verbände haben mit dieser Arbeit begonnen und die Personalentwicklung mittlerweile fest in ihren Leitbildern und Unternehmensgrundsätzen verankert.

Beispiel Deutsches Rote Kreuz:« Zu unserer Aufgabe als Führungskräfte gehört auch die gezielte Auswahl sowie die systematische Qualifizierung unserer Helfer und Mitarbeiter im Rahmen unserer Personalentwicklung ...« (Das DRK-Leitbild, Bonn 1995)

Beispiel Arbeiterwohlfahrt: »Um die Qualität der Dienstleistungen zu steigern, Mitarbeiter und Mitarbeiterinnen zu motivieren und ihnen Perspektiven für das berufliche Weiterkommen zu sichern, ist die persönliche und berufliche Entwicklung durch kontinuierliche Fortbildung zu fördern ...« (Grundsatzprogramm der Arbeiterwohlfahrt, Bonn 1998/99)

Wie die Großen, so werden auch die kleinen Einrichtungen nicht umhin kommen, sich mit diesen grundsätzlichen Fragen zu beschäftigen. Und natürlich braucht das seine Zeit. Dennoch ist es nicht sinnvoll darauf zu warten, bis ein Leitbild formuliert und eine Unternehmens- strategie festgelegt wurde. Mit Personalentwicklung kann man sofort beginnen; es können ja kleine Schritte sein (wie ein Beispiel im übernächsten Kapitel zeigt).

\section{„Bei uns ist doch alles anders! Oder nicht?»}

Sicherlich läuft Personalentwicklung in einem zweigruppigen Kindergarten anders als bei einem Versicherungskonzern, aber eines haben sie doch gemeinsam: Hier wie dort geht es um die Mitarbeiterinnen und Mitarbeiter. Es geht um das Wachstum ihrer Persönlichkeit, ihres Könnens und Wissens. Dazu gibt es einen interessanten Ansatz von Michael Paschen (Paschen, M: Instrumente der Personalentwicklung, Books on Demand, 2004): »Personalentwicklung geschieht dort, wo Aufgaben und Herausforderungen geeignet sind, um Wachstumsprozesse auszulösen und wo das Unternehmen bereit ist, die Rahmenbedingungen dafür zu schaffen, dass solche Wachstumsprozesse gefördert werden.«Und das können kleine Einrichtungen ebenso wie große Konzerne. Alle gängigen Personalentwicklungsmaßnahmen kommen grundsätzlich auch für soziale Einrichtungen in Frage (vgl. Abb. 1).
Jede Maßnahme kann so an die Erfordernisse der Einrichtung angepasst werden, dass sie realisierbar und mit dem Ziel der Einrichtung vereinbar ist und außerdem die Motivation der Mitarbeitenden erhält und steigert. Am Beispiel »Einführung neuer Mitarbeiterinnen und Mitarbeiter « heißt dies, dass ein Autohersteller vielleicht für seine neuen Mitarbeiter ein Tagesseminar inklusive zweistündiger Werksführung durchführt, in einem kleinen Kindergarten wird die Einführung eher mit einem kleinen Rundgang mit anschließendem Kennenlerntreffen der Kollegenschaft beginnen. Beides ist richtig und führt zum Ziel. Im Folgenden wird unter anderem das Beispiel wieder aufgegriffen.

\section{Wie man es machen kann}

Beim genauen Hinschauen wird deutlich werden, dass in jeder Einrichtung bereits einiges für die fachliche und persönliche Entwicklung der Mitarbeiterinnen und Mitarbeiter getan wird, meist allerdings nicht strukturiert und 
regelhaft. Um aufbauend auf dem bereits Vorhandenen zu einer systematischen Personalentwicklung zu kommen, sind meines Erachtens sechs Schritte sinnvoll.

\section{Schritt eins: Klärung der Zuständigkeit}

Es muss entschieden werden, wer für die Personalentwicklung zuständig ist. In großen Unternehmen werden zum Zwecke der Personalentwicklung zentrale Abteilungen geschaffen, um die Konzeption, Durchführung und Evaluation von Personalentwicklungsmaßnahmen zu bündeln. Diesen Ansatz sollte man in kleinen Einrichtungen übernehmen: Man braucht eine Person (oder zwei), die für dieses Aufgabenfeld verantwortlich ist. Dies sollte in jedem Fall ein Vertreter des Trägers sein, beispielsweise der Geschäftsführer oder ein Vorstandsmitglied - denn Personalentwicklung ist Managementaufgabe. Bei der Aufgabenzuordnung im Leitungsgremium ist Personalentwicklung fest als Teil des Tagesgeschäftes mit einzuplanen. Darüber hinaus sind natürlich die Regelungen zur Mitbestimmung zu beachten.

\section{Schritt zwei: Brainstorming}

Zunächst gilt es, einen Überblick über den Stand der Personalentwicklung in der Einrichtung zu erhalten und eine Richtung für die weitere Entwicklung zu erarbeiten. Für diese Aufgabe eignet sich ein gezieltes Brainstorming. Um zielgerichtet und zügig arbeiten zu können, bietet es sich an, einen moderierten Workshop mit einem Teilnehmerkreis aus dem Vorstand, der Geschäftsleitung und Mitarbeitervertretern durchzuführen.

So ein Brainstorming hat drei Vorteile: Man sieht, welche Personalentwicklungsmaßnahmen bereits verwirklicht werden - und das ermuntert zum Weiterarbeiten,

\begin{tabular}{|l|l|}
\hline $\begin{array}{l}\text { Mögliche Fragestellung } \\
\text { im Brainstorming }\end{array}$ & Mögliche Antworten \\
\hline $\begin{array}{l}\text { Was wollen wir mit syste- } \\
\text { matischer Personalent- } \\
\text { wicklung erreichen? }\end{array}$ & $\begin{array}{l}\text { Erhalt der Einrichtung, Verbesserung der Qua- } \\
\text { lität, Motivation der Mitarbeiter, Einheitlich- } \\
\text { keit der Mitarbeiterförderung ... }\end{array}$ \\
\hline $\begin{array}{l}\text { Was haben wir schon? } \\
\text { Regelmäßige Mitarbeitergespräche, externe } \\
\text { Fachseminare, Coachings ... }\end{array}$ \\
\hline $\begin{array}{l}\text { Wainführen? } \\
\text { kunft entwickeln? }\end{array}$ & $\begin{array}{l}\text { Einarbeitungsprogramm für neue Mitarbeiter, } \\
\text { Projektarbeit, Verhaltenstrainings, Vorschlags- } \\
\text { wesen ... }\end{array}$ \\
\hline $\begin{array}{l}\text { Was muss noch diskutiert } \\
\text { werden? }\end{array}$ & Job Rotation, Teamtrainings ... \\
\hline
\end{tabular}

Abb. 2: Mit einem Brainstorming können erste Grundlagen für ein Konzept zur systematischen Personalentwicklung gelegt werden.
Ideen gehen nicht verloren und die Sammlung kann jederzeit ergänzt werden (vgl. Abb. 2).

Das Ergebnis des Workshops kann eine Sammlung der gesamten Personalentwicklungsmaßnahmen sein, einschließlich Zeitplan zur Einführung oder Weiterbearbeitung (vgl. Abb. 3).

\section{Schritt drei: Personalentwicklungskonzept}

Nach meiner Erfahrung ist das Thema Personalentwicklung sowohl in großen als auch in kleinen Unternehmen oft emotionsgeladen. Mancher Mitarbeiter fühlt sich gegängelt,

\section{»Personalentwicklung muss eingebettet sein in Strategie, Struktur und Kultur des Unternehmens «}

weil er oft zu Schulungen geschickt wird, ein anderer vermisst die Förderung und die damit verbundene Wertschätzung. Oft wird Personalentwicklung als willkürlich erlebt.

Ein schriftliches und allen zugängliches Personalentwicklungskonzept wirkt dem entgegen. Es schafft Transparenz und Verbindlichkeit und motiviert. Nun kann man mit der Formulierung und Veröffentlichung eines solchen Konzeptes warten, bis alle geplanten Maßnahmen schließlich in die Tat umgesetzt wurden. Dies kann allerdings Jahre in Anspruch nehmen. Ich plädiere daher dafür, schrittweise vorzugehen: Es werden in dem Konzept zunächst nur die bereits verwirklichten Personalentwicklungsmaßnahmen beschrieben und dann nach und nach die neu eingeführten Maßnahmen ergänzt.

So ein Konzept muss keine Hochglanzbroschüre sein, auch wenn es durchaus sinnvoll sein kann, bei der Formulierung externe Hilfe in Anspruch zu nehmen. In dem Konzept sollten für jede bereits eingeführte Personalentwicklungsmaßnahme fünf Aspekte beschrieben sein: Ziele der Maßnahme; Inhalte; beteiligte Personen (z. B. Vorgesetzter und Mitarbeiter); Vorgehensweise (z. B. Antrag durch den Mitarbeiter, Entsendung durch die Einrichtungsleitung etc.); Regelungen zur Arbeitszeit und Kosten.

\section{Schritt vier: kurzfristige Ein- führung neuer Maßnahmen}

Grundlage für die Entwicklung und Einführung neuer Maßnahmen sind die Ergebnisse aus dem Brainstorming.

Jetzt geht es vor allem darum, die Aspekte Ziele, Inhalte und weitere Vorgehensweise, die im Brainstorming bereits grob umrissen wurden, mit 


\begin{tabular}{|c|c|c|c|c|c|c|c|}
\hline $\begin{array}{l}\text { Personalent- } \\
\text { wicklungs- } \\
\text { maßnahme }\end{array}$ & $\begin{array}{l}\text { Ziele der } \\
\text { Maßnahme }\end{array}$ & $\begin{array}{l}\text { Inhalte der } \\
\text { Maßnahme }\end{array}$ & Stand & $\begin{array}{l}\text { Weitere Vor- } \\
\text { gehensweise }\end{array}$ & $\begin{array}{l}\text { Realisierung } \\
\text { bis wann }\end{array}$ & $\begin{array}{l}\text { verantwort- } \\
\text { lich }\end{array}$ & Kosten \\
\hline $\begin{array}{l}\text { Regelmäßige } \\
\text { Mitarbeiterge- } \\
\text { spräche }\end{array}$ & $\begin{array}{l}\text { 1. Standortbe- } \\
\text { stimmung } \\
\text { 2. Leistungs- } \\
\text { vereinbarun- } \\
\text { gen } \\
\text { 3. Motivation }\end{array}$ & $\begin{array}{l}\text { Jährlicher } \\
\text { Austausch - } \\
\text { zwischen Vor- } \\
\text { gesetztem und } \\
\text { Mitarbeiter zu } \\
\text { den Themen } \\
\text { Arbeitsbedin- } \\
\text { gungen, Ziele, } \\
\text { Zusammenar- } \\
\text { beit }\end{array}$ & $\begin{array}{l}\text { Wird bereits } \\
\text { durchgeführt, } \\
\text { allerdings } \\
\text { nicht struktu- } \\
\text { riert }\end{array}$ & $\begin{array}{l}\text { Entwicklung } \\
\text { von Leitfragen } \\
\text { für strukturier- } \\
\text { te Gespräche }\end{array}$ & 05/09 & $\begin{array}{l}\text { Herr X und } \\
\text { Frau Y }\end{array}$ & \\
\hline \multicolumn{8}{|l|}{$\ldots$} \\
\hline $\begin{array}{l}\text { Einarbeitungs- } \\
\text { programm }\end{array}$ & $\begin{array}{l}\text { 1. Vermittlung } \\
\text { von Fach- } \\
\text { wissen } \\
\text { 2. Vermittlung } \\
\text { der Werte } \\
\text { der Einrich- } \\
\text { tung } \\
\text { 3. Motivation } \\
\text { 4. Nutzen des } \\
\text { Ideenpoten- } \\
\text { zials neuer } \\
\text { Mitarbeiter }\end{array}$ & $\begin{array}{l}\text { Einführung } \\
\text { neuer Mitar- } \\
\text { beiter vom Be- } \\
\text { werbungsver- } \\
\text { fahren bis zum } \\
\text { Ende der Pro- } \\
\text { bezeit }\end{array}$ & $\begin{array}{l}\text { Noch keine re- } \\
\text { gelhafte Vorge- } \\
\text { hensweise }\end{array}$ & $\begin{array}{l}\text { Erarbeitung } \\
\text { der einzelnen } \\
\text { Bausteine in } \\
\text { einer Arbeits- } \\
\text { gruppe, Unter- } \\
\text { stützung durch } \\
\text { externe Mode- } \\
\text { ration }\end{array}$ & $\begin{array}{l}\text { Präsentation } \\
\text { der Ergebnisse } \\
\text { in } 07 / 09 \\
\text { Umsetzung ab } \\
08 / 09\end{array}$ & Arbeitsgruppe & $\begin{array}{l}\text { Kosten für die } \\
\text { Moderation }\end{array}$ \\
\hline
\end{tabular}

Abb. 3: Aufbauend auf dem Brainstorming kann ein Gesamtkonzept mit Zeitplan zur systematischen Personalentwicklung aufgestellt werden.

\begin{tabular}{|c|c|c|}
\hline Ziele der Maßnahme & Inhalte der Maßnahme & Weitere Vorgehensweise \\
\hline $\begin{array}{l}\text { Vermittlung von Fachwissen } \\
\text { - Abläufe am Arbeitsplatz } \\
\text { - Abläufe in der Einrichtung } \\
\text { - Wissenslücken schließen }\end{array}$ & $\begin{array}{l}\text { - Unterweisungsgespräch mit dem Vorge- } \\
\text { setzten } \\
\text { - Kennenlernen aller Kollegen } \\
\text { - stunden-/tageweise Hospitation bei Kolle- } \\
\text { gen } \\
\text { - Checkliste für die Einarbeitung } \\
\text { - } \ldots\end{array}$ & $\begin{array}{l}\text { - Entwurf einer Checkliste für jeden Ar- } \\
\text { beitsplatz } \\
\text { - . . }\end{array}$ \\
\hline $\begin{array}{l}\text { Vermittlung der Werte und Leitlinien } \\
\text { der Einrichtung } \\
\text { - in der Darstellung nach außen } \\
\text { - im Umgang mit Kunden } \\
\text { - im Umgang mit Kollegen und Vor- } \\
\text { gesetzten }\end{array}$ & $\begin{array}{l}\text { - regelmäßige Gespräche mit Vorgesetzten } \\
\text { und/oder Mentoren } \\
\text { - . . }\end{array}$ & $\begin{array}{l}-\ldots \\
-\ldots\end{array}$ \\
\hline $\begin{array}{l}\text { Motivation } \\
\text { - Schaffen einer positiven Grundein- } \\
\text { stellung beim Bewerber } \\
\text { - neue Mitarbeiter sollen sich will- } \\
\text { kommen fühlen } \\
\text { - neue Mitarbeiter sollen die Aufga- } \\
\text { ben als bewältigbar erleben }\end{array}$ & $\begin{array}{l}\text { - Informationsmaterial über die Einrich- } \\
\text { tung für den Bewerber } \\
\text { - Schriftwechsel mit dem Bewerber } \\
\text { - strukturiertes, professionell geführtes Be- } \\
\text { werbergespräch } \\
\text { - Vorbereitung des Arbeitsplatzes } \\
\text { - Infomappe für neue Mitarbeiter } \\
\text { - Feedbackgespräche mit Vorgesetzten } \\
\text { und/oder Mentoren } \\
\text { - ... }\end{array}$ & $\begin{array}{l}\text { - Zusammenstellung einer Infomappe } \\
\text { - Überprüfung der Standardbriefe } \\
\text { - Formulierung einer Anleitung für Bewer- } \\
\text { bergespräche } \\
\text { - Formulierung von Leitfragen für ein } \\
\text { Feedbackgespräch } \\
\text { - ... }\end{array}$ \\
\hline $\begin{array}{l}\text { Nutzen des Ideenpotenzials } \\
\text { - Schaffen einer vertrauensvollen At- } \\
\text { mosphäre } \\
\text { - Möglichkeiten des Austausches } \\
\text { schaffen }\end{array}$ & $\begin{array}{l}\text { - regelmäßige Gespräche mit Vorgesetzten } \\
\text { und/oder Mentoren } \\
\text { - . . }\end{array}$ & $\begin{array}{l}-\ldots \\
-\ldots\end{array}$ \\
\hline
\end{tabular}


Leben zu füllen. Auch in diesem Schritt bietet sich ein moderierter Workshop an, entweder mit dem gleichen Teilnehmerkreis wie beim Brainstorming oder mit einer kleineren Arbeitsgruppe. Das Beispiel »Einführung eines Einarbeitungsprogramms für neue Mitarbeiterinnen und Mitarbeiter « zeigt, wie Ergebnisse des Workshops aussehen können (vgl. Abb. 4).

Sicherlich wird es so sein, dass viele Elemente schon in der Einrichtung praktiziert werden. Und so muss man nur schauen, an welcher Stelle noch etwas verbessert oder systematisiert werden kann. Nach diesem Muster können alle geplanten Maßnahmen einzeln betrachtet werden.

\section{Schritt fünf: Weiterverfolgung der langfristig ge- planten Maßnahmen}

Parallel oder zeitlich nach der Einführung der kurzfristig geplanten neuen Maßnahmen wird an den langfristig zu planenden Maßnahmen gearbeitet. Je nach den zur Verfügung stehenden Ressourcen kann dies mehrere Jahre in Anspruch nehmen. Die Vorgehensweise gleicht der im vierten Schritt.

\section{Schritt sechs: Jährliche Überprüfung}

Wie schon erwähnt: Personalentwicklung existiert nicht im luftleeren Raum, sondern ist immer eingebettet in die Strategie, Struktur und Kultur des Unternehmens. Und natürlich ist sie auch ein Teil des Arbeitsalltags, sowohl für Mitarbeitende als auch für Führungskräfte und Geschäftsleitung. Um hier immer wieder einen Abgleich zu schaffen und wichtige Aspekte zu überprüfen - Passt unsere Personalentwicklung noch zu unserer Strategie? Sind wir zufrieden mit unseren bisherigen Ergebnissen? Wie machen wir weiter? - ist es sinnvoll, etwa einmal jährlich Vertreter von Vorstand, Geschäftsleitung und Mitarbeitenden zu einem Strategie-Workshop zusammen zu holen. Dieser Austausch sorgt dafür, dass die Arbeit an der Personalentwicklung nicht in Vergessenheit gerät und ermöglicht es, die gemeinsam gewählte Strategie zu bestätigen oder auch neu zu formulieren.

\section{Resümee}

Drei Erkenntnisse sollte der Beitrag vermitteln: Systematische Personalentwicklung ist erstens auch in der Sozialwirtschaft notwendig. Systematische Personalentwicklung ist zweitens auch in kleinen Organisationen machbar. Maßnahmen und Instrumente aus der Wirtschaft können drittens auch für soziale Betriebe geeignet sein.

Die systematische Entwicklung und Förderung der Mitarbeiterinnen und Mitarbeiter als Beitrag zum langfristigem Erhalt und Erfolg der Einrichtung ist also nicht nur den Großen vorbehalten, sondern auch Kleine können diesen Weg gehen. Und jeder Weg beginnt mit einem ersten kleinen Schritt.

\section{Soziales Europa}

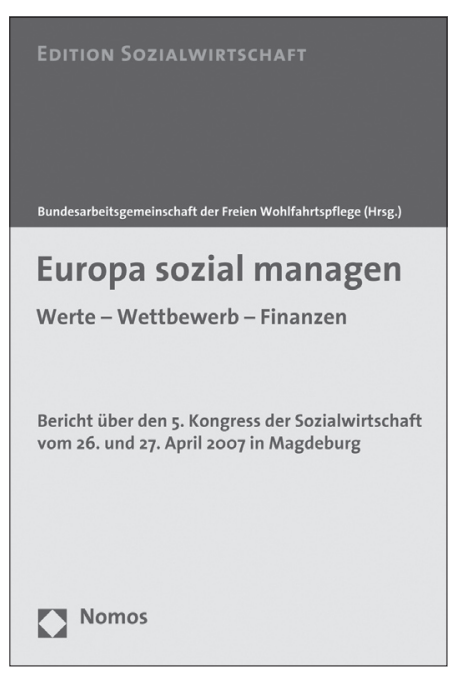

\section{Europa sozial managen}

Werte - Wettbewerb - Finanzen

Bericht über den 5. Kongress der Sozialwirtschaft vom 26. und 27. April 2007 in Magdeburg

Herausgegeben von der Bundesarbeitsgemeinschaft der Freien Wohlfahrtspflege (BAGFW)

2008, 193 S., brosch., 39,- €, ISBN 978-3-8329-3190-2

(Edition Sozialwirtschaft, $B d .24)$

Zusammen mit europäischen Partnern können soziale Dienste und Einrichtungen in Deutschland ihre Arbeit optimieren. Die deutsche Sozialwirtschaft kann, wenn sie sich auf internationale Kooperationen einlässt, mit einer europäischen Dividende rechnen. Das war die Botschaft des 5. Kongresses der Sozialwirtschaft, der am 26. und 27. April 2007 in Magdeburg stattfand, zum Thema »Europa sozial managen - Werte, Wettbewerb, Finanzen«. Veranstalter dieses alle zwei Jahre stattfindenden Branchentreffs sind die Bundesarbeitsgemeinschaft der Freien Wohlfahrtspflege, die Bank für Sozialwirtschaft und die Nomos Verlagsgesellschaft.

In zwei Plenumsveranstaltungen und sechs Foren diskutierten Führungskräfte mit Referenten aus ganz Europa die konkreten Auswirkungen der europäischen Einigung auf die Sozialwirtschaft - von den Folgen des europäischen Vergaberechts auf deutsche Leistungserbringer bis zu den Auswirkungen der Dienstleistungsrichtlinie für Import und Export sozialer Dienstleistungen.

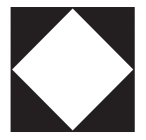

Nomos

Bitte bestellen Sie im Buchhandel oder versandkostenfrei unter $\downarrow$ www.nomos-shop.de 